\title{
A Scheme of Mobility Management in UDN Based on PPP
}

\author{
Junxuan Wang ${ }^{1}$, Ruixin $\mathrm{Li}^{1}$ and Tianxiang $\mathrm{Ji}^{1}$ \\ Shaanxi Key Laboratory of Information Communication Network and Security, \\ $X i^{\prime}$ an University of Post and Telecommunications, Xi'an 710100, China \\ 1842930104@qq.com
}

\begin{abstract}
$5 G$ networks will adopt an Ultra Dense Network architecture that can support higher speed and provide lower latency. In some hotspots, small base stations need to be deployed on a large scale in order to meet the demands of massive data communications. In this ultra-dense network environment, traditional mobility management algorithms have been unable to adapt to the future development of the network. How to address the problem of mobility management in the ultra-dense network environment has become a huge challenge for the future development of the network. This paper proposes a mobility management scheme based on precise point positioning. Using precise point positioning technology to get the user's precise location and current speed, thereby predicting the user's location at the next moment, and making handover decisions based on this result. Simulation results demonstrate that compared with the traditional scheme. The scheme significantly reduces the Ping-Pong effect and the average switching delay.
\end{abstract}

Keywords: Ultra Dense Network(UDN), mobility management, precise point positioning $(P P P)$, prediction

\section{Introduction}

The next generation of Wireless Communications is challenged to enhance users' experience, support new services, and satisfy the ever-increasing mobile user population and their traffic demands. In order to solve the future network data traffic increased by 1000 times, Ultra Dense Network is regarded as the most promising key technology of $5 \mathrm{G}[1]$.

In UDN, with the reduction of the power, most of the microcell base station coverage will be in 50 200m. With the development of technology, it will even shrink to about 10m.However, comparing with traditional cellular architecture, this architecture makes handover more complex when the user moves out the microcell service area in a short time[2].In this scenario, the crucial problem in mobility management is how to for the user to select the best serving base station.

In the traditional scheme, user mobility management is mainly based on the user location and speed estimation of the cellular network. Yeung and Nanda proposed estimating the speed based on the number of cells that mobile terminals pass in a long interval, and use this speed to perform layer selection of multi-layer cellular structures [3]. Sung and Wong use the first handover time to get the speed [4]. Wann and Chen proposed using the cellular network positioning technology to determine the location of the mobile terminal, and then using the Kalman filter to process the position information sequence, thus obtaining an accurate estimation of speed and position [5]. These traditional mobility management schemes are simple to implement, but they are very inaccurate and require a long estimation time, so they cannot solve the current problems in the 5G system. To address these issues, various mobility management schemes have been proposed to

Received (May 15, 2018), Review Result (July 25, 2018), Accepted (August 21, 2018) 
improve the system performance. A formula between handover and velocity was proposed for the handover trigger based on the assumption that the velocity is fixed when the user is moving [6]. However, the user usually takes the random walk model in the real environment, where the user moves no certain velocity. Meanwhile, the user's velocity is estimated by GPS standard single point positioning, in which the error could be large. It is proposed to solve the problem by using Machine Learning(ML) algorithms, such as Hidden Markov Model(HMM) [7, 8] and Support Vector Machine(SVM) [9]. With these methods, it is needed the user's historical movement data having a trend, and the largescale training can't use in mobile computing environment.

To solve the mobility management, we proposed a mobility management scheme based on the Global Navigation Satellite System(GNSS) Precise Point Positioning. In the proposed scheme, the user's PPP measurement position and velocity are used to predict the next position. After that, handover trigger decisions will be made with the prediction values, which would reduce the unnecessary handover and enhance the system performance.

The remainder of the paper is organized as follows. The proposed mobility management scheme is given in Section II. The simulation results are presented and analyzed in Section III. Finally, we draw a conclusion and summarize our work in Section VI.

\section{Precise Point Positioning Based Mobility Management Scheme}

\subsection{Network Structure}

In an Ultra Dense Network, a macrocell contains a series of microcells $B S_{1}, B S_{2}, \cdots, B S_{N}$. The macrocell still adopts a traditional cellular hexagonal structure. The microcells are assumed to be distributed over a two-dimensional grid geographic area. As shown in Figure 1, each grid and its center represent the coverage of the microcell and the location of the base station. In this area, we assume that the UE is initially connected to $B S_{i}$, and its geographical coordinates at time $\mathrm{t}$ are denoted by $(x(t), y(t))$. In the communication process, handover occurs when the UE leaves the $B S_{i}$ covered area and enters a $B S_{j}$ covered area. The number of neighbor microcells is determined by the density of microcells.

\subsection{Precise Point Positioning}

Precise point positioning uses the precise ephemeris and precise clock products provided by the International GPS Service (IGS) to position and solve the pseudorange and carrier phase observations collected by a single receiver, which can reach $2-4 \mathrm{~cm}$ level positioning accuracy. With more and more Global Navigation Satellite System satellites being in view and terrestrial tracking stations increasing precise point positioning has become a very hot research topic[10,11].

2.2.1. GNSS Original Observation Equation: In GNSS, the most basic observations are pseudorange and carrier phase. The original GNSS observation equations are written as[12]: 


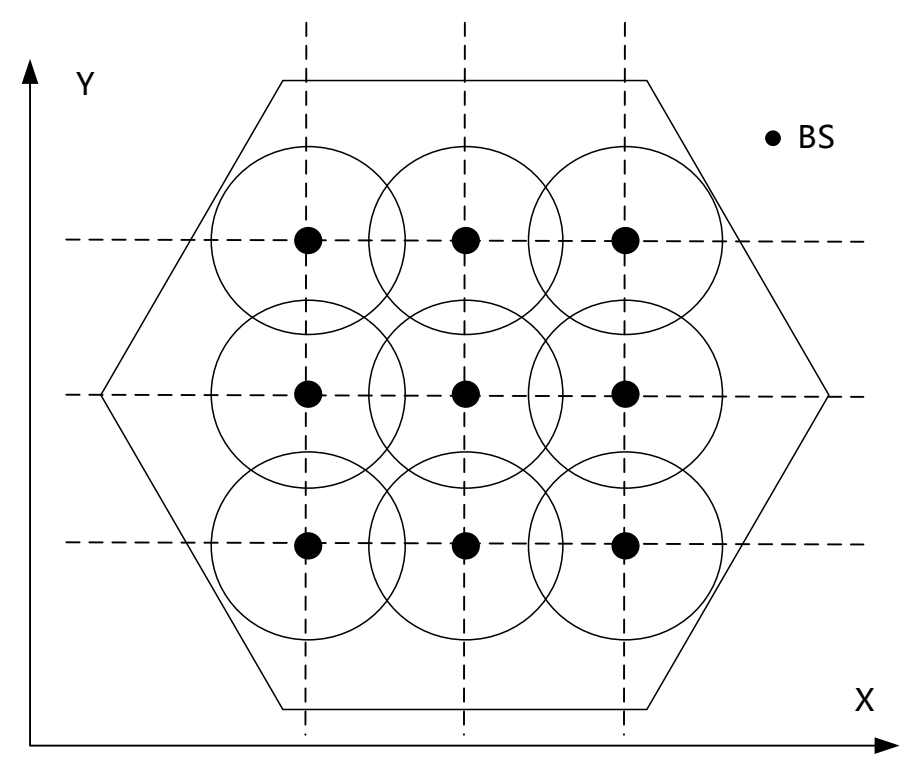

Figure 1. Network Structure

$P_{i}=\rho+c\left(d t_{r}-d t^{s}\right)+d_{\text {trop }}+d_{\text {ion }}+d_{\text {other }}+\varepsilon_{P}$

$L_{i}=\rho+c\left(d t_{r}-d t^{s}\right)+d_{\text {trop }}-d_{\text {ion }}+\lambda_{i} N_{i}+d_{\text {other }}+\varepsilon_{L}$

Where:

$P_{i}, L_{i}$ are the pseudorange and carrier phase observations at the frequency i in $[\mathrm{m}]$, respectively;

$d t_{r}, d t^{s}$ are the satellite and receiver clock offset in [s], respectively;

$\rho$ is the geometric distance from the satellite to the receiver antenna phase center in $[\mathrm{m}]$; $\mathrm{c}$ is the light speed in vacuum in $[\mathrm{m} / \mathrm{s}]$;

$d_{\text {trop }}, \quad d_{\text {ion }}$ are the tropospheric delay and the ionosphere delay in[m];

$\lambda_{i}$ is the carrier wavelength at the frequency $\mathrm{i}$ in $[\mathrm{m}]$;

$N_{i}$ is integer ambiguity at the frequency $\mathrm{i}$ in [cycle];

$d_{\text {other }}$ are the Earth tide error, pole tide error, satellite and receiver antenna phase center offset, multipath effects, and other errors in [m];

$\varepsilon_{P}, \varepsilon_{L}$ are the measurement noise in pseudorange and carrier phase observations in [m],respectively.

All the original information is preserved in the original observations, and the observation equations of different satellites are almost irrelevant. However, each of the above errors must be eliminated or weakened by the model or estimated as an unknown parameter. Otherwise, it will affect the positioning accuracy of the precise point positioning.

2.2.2. Ionosphere-free Linear Combination Observation: In the equations (1) and (2),some error terms are related to the frequency of observations, such as ionosphere delay Due to the dispersion characteristics of the ionosphere delay, a linear combination of ionosphere-free observations[9] can be made using raw observations of two frequencies $f_{1}$ and $f_{2}$, which eliminate the effects of ionosphere delay The pseudorange and carrier phase measurements of the ionosphere-free linear combination observations are as follows: 


$$
\begin{aligned}
& P_{I F}=\frac{1}{f_{1}^{2}-f_{2}^{2}}\left(f_{1}^{2} P_{1}-f_{2}^{2} P_{2}\right) \\
& L_{I F}=\frac{1}{f_{1}^{2}-f_{2}^{2}}\left(f_{1}^{2} L_{1}-f_{2}^{2} L_{2}\right)
\end{aligned}
$$

Precise point positioning usually uses ionosphere-free pseudorange and carrier phase measurement as the position observation model, eliminating the influence of ionosphere first-order terms.

2.2.3. Error Processing Method: Compared with standard single-point positioning using broadcast ephemeris and clock, precise point positioning uses precise ephemeris and precise clock products provided by IGS. The satellite orbit error can be reduced to about $2 \mathrm{~cm}$, and the satellite clock error can be reduced to $0.3 \mathrm{~ns}$, which greatly weakens the influence of satellite orbit error and satellite clock error on positioning. For tropospheric delay, the Saastamoinen model[13] is generally used for correction. The multipath error needs to be corrected by extending the observation time, wavelet analysis, and stellar day filtering. The Earth tide error and pole tide error need to be corrected by corresponding models, and the residual error after correction is within a few millimeters.

\subsection{Path Loss Model}

In this paper, according to the $\mathrm{B} 1$ and $\mathrm{C} 2$ scenario configuration of WINNERII[14], the microcell path loss model and the macrocell path loss model are written as:

$$
\begin{aligned}
& P L_{\text {macro }}(i, x)=26 \log _{10}(x)+20 \log _{10}\left(\frac{f_{\text {macro }}}{5.0}\right)+39+\varepsilon(i, x) \\
& P L_{\text {micro }}(m, y)=22.7 \log _{10}(y)+20 \log _{10}\left(\frac{f_{\text {micro }}}{5.0}\right)+41+\varepsilon(m, y)
\end{aligned}
$$

Where:

$P L_{\text {macro }}, P L_{\text {micro }}$ are the path loss of macrocell and microcell in $[\mathrm{dB}]$;

$i, m$ are the macrocell base station $\mathrm{i}$ and microcell base station $\mathrm{m}$;

$x, y$ are the distance from UE to the macrocell base station i, the distance from UE to the microcell base station $\mathrm{m}$ in $[\mathrm{m}]$, respectively;

$f_{\text {macro }}, f_{\text {micro }}$ are the carrier frequency of macrocell and microcell in [GHz], respectively;

$\varepsilon(i, x), \varepsilon(m, y)$ are the shadow fading and obey Gaussian distribution with zero mean and standard deviation $\sigma$.

Based on the path loss model, the UE received signal power can be derived as follows:

$$
\begin{aligned}
& P r_{\text {macro }}(i, x)=P t_{\text {macro }}-P L_{\text {macro }}(i, x) \\
& P r_{\text {micro }}(m, y)=P t_{\text {micro }}-P L_{\text {micro }}(m, y)
\end{aligned}
$$

Where:

$P r_{\text {macro }}, P r_{\text {micro }}$ are the receives signal power of UE in the macrocell and microcell in $[\mathrm{dBm}]$, respectively;

$P t_{\text {macro }}, P t_{\text {micro }}$ are the transmit signal power of macrocell and microcell in $[\mathrm{dBm}]$, respectively.

Taking into account the co-channel interference between cells and inter-frequency interference, the interference of macrocell and microcell can be obtained as: 
$I_{i}\left(\begin{array}{ll}\text { or } & I_{m}\end{array}\right)=10 \log _{10}\left[\sum_{k=1}^{N_{\text {nei } e N B}} 10^{\frac{\operatorname{Pr}(k, x)}{10}}\right]$

Where:

$I_{i}, I_{m}$ are the interference of macrocell $\mathrm{i}$ and microcell $\mathrm{m}$ in $[\mathrm{dBm}]$, respectively;

$N_{n e i-e N B}$ is the neighbor cells, including macrocell and microcell.

The signal-to-interference ratio (SIR) of UE received signal quality of the macrocell or the microcell can be expressed as:

$$
\begin{aligned}
& \operatorname{SIR}_{\text {macro }}(i, x)=P r_{\text {macro }}(i, x)-I_{i} \\
& \operatorname{SIR}_{\text {micro }}(m, y)=P r_{\text {micro }}(m, y)-I_{m}
\end{aligned}
$$

Where:

$\operatorname{SIR}_{\text {macro }}(i, x), \operatorname{SIR}_{\text {micro }}(m, y)$ are the SIR of macrocell and microcell in [dB].

\subsection{Handover Decision Scheme}

This paper mainly considers the problem of microcell mobility management in Ultra Dense Network hotspots. It is assumed that the SIR is an indicator of handover trigger. Figure 2 describes the handover trigger decision-making procedure. Precise point positioning is used to measure the current actual position and speed of UE, and calculate the UE's SIR. Then estimates the UE's position of the next moment according to the direction of the speed. Simultaneously, the SIR of UE in the next moment is calculated. The difference is used to compare with the threshold. If reached the threshold, the target cell prepares the corresponding resources for UE handover in advance and waits for UE to access the next time; if the precise point positioning measurement result of the next moment matches the prediction, it means that the user has entered the target cell, then the user is immediately allowed to access, otherwise prediction error, the target cell releases the prepared resources.

\section{Simulation and Performance Analysis}

\subsection{Precise Point Positioning Accuracy}

The experiment selected the observation data of the day of April 18, 2018 in Xi'an and the precise ephemeris and precise clock products provided by IGS, and used the RTKLIB software packages developed by T. Takasu of Tokyo University of Marine Science and Technology to solve the data[15]. The solutions are shown in Figure 3. It can be seen from the results that in the station center coordinate system, the positioning errors in the $\mathrm{E}, \mathrm{N}, \mathrm{U}$ directions do not exceed $5 \mathrm{~cm}$.Compared with the standard single-point positioning accuracy, the precise point positioning accuracy is greatly improved. 


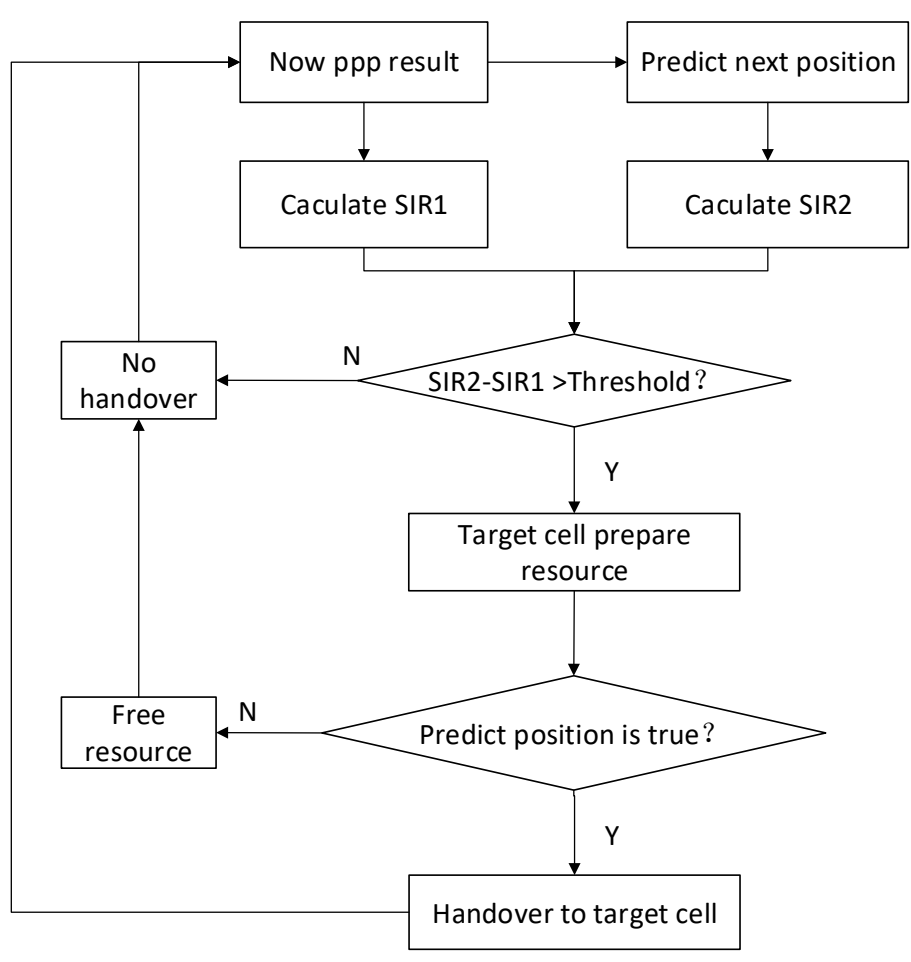

Figure 2. Handover Trigger Decision-making Flowchart

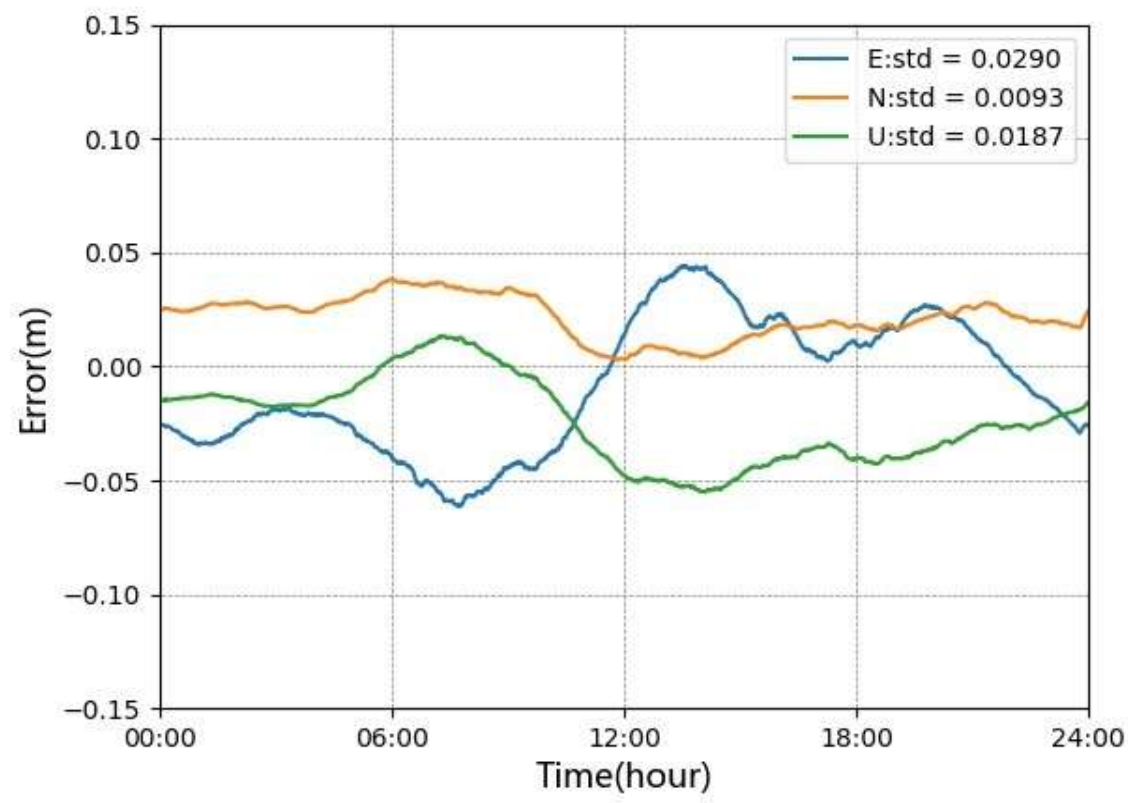

Figure 3. Precise Point Positioning Error

\subsection{Handover Probability}

The new mobility management scheme has been proposed in the section III of this paper. If the SIR of the target microcell is higher than the UE current connecting microcell $\Gamma \mathrm{dB}$, handover from the current cell to the target cell will occur. Therefore, the handover probability from the source microcell $i$ to the target microcell $\mathrm{j}$ is as follows: 


$$
\begin{aligned}
P\left(i, j, x_{i}, x_{j}\right)_{H O}= & P\left[\operatorname{SIR}\left(j, x_{j}\right)-\operatorname{SIR}\left(i, x_{i}\right)>\Gamma\right] \\
= & P\left[\left(\operatorname{Pr}\left(j, x_{j}\right)-\mathrm{I}_{j}\right)-\left(\operatorname{Pr}\left(i, x_{i}\right)-\mathrm{I}_{i}\right)>\Gamma\right] \\
= & P\left[\operatorname{PL}\left(i, x_{i}\right)-\operatorname{PL}\left(j, x_{j}\right)+\mathrm{I}_{i}-\mathrm{I}_{j}>\Gamma\right] \\
= & P\left[\varepsilon\left(i, x_{i}\right)-\varepsilon\left(j, x_{j}\right)>\Gamma+\operatorname{Alog}_{10}\left(x_{j} / x_{i}\right)\right. \\
& \left.\quad+\mathrm{I}_{j}-\mathrm{I}_{i}\right] \\
= & \mathrm{Q}\left[\frac{\Gamma+\operatorname{Alog}_{10}\left(x_{j} / x_{i}\right)+\mathrm{I}_{j}-\mathrm{I}_{i}}{\sigma\left(i, x_{i}\right)-\sigma\left(j, x_{j}\right)}\right]
\end{aligned}
$$

Where:

$P\left(i, j, x_{i}, x_{j}\right)_{H O}$ is handover probability from microcell i to microcell $\mathrm{j}$;

$x_{i}, x_{j}$ are the distance from $\mathrm{UE}$ to the microcell $\mathrm{i}$ base station and microcell $\mathrm{j}$ base station, respectively;

A is the microcell path Loss parameters in section III;

$\varepsilon\left(i, x_{i}\right), \varepsilon\left(j, x_{j}\right)$ are the shadow fading, $\varepsilon\left(i, x_{i}\right) \sim N\left(0, \sigma^{2}\left(i, x_{i}\right)\right)$ and $\varepsilon\left(\mathrm{j}, x_{j}\right) \sim N\left(0, \sigma^{2}\left(j, x_{j}\right)\right)$.

Using the proposed scheme in this paper, we need to predict the distance from the UE to the target microcell $x_{j}$, define the positioning error factor $\delta$ is as follows:

$\delta=\frac{e}{R}$

Where:

$\mathrm{e}$ is the positioning inherent error;

$\mathrm{R}$ is the microcell radius.

The relationship between the predicted distance from the UE and the target microcell $\hat{x}_{j}$ and real distance $x_{j}$ is written as :

$\hat{x}_{j}=(1-\delta) x_{j}$

Based on equations (12) and (14), we can get the relationship between the handover probability using precise point positioning and the handover probability using standard single point positioning is as follows :

$$
P\left(i, j, x_{i}, x_{j}\right)_{H O}<P\left(i, j, x_{i}, \hat{x}_{j, p p p}\right)_{H O}<P\left(i, j, x_{i}, \hat{x}_{j, \text { tradition }}\right)_{H O}
$$

Where:

$P\left(i, j, x_{i}, x_{j}\right)_{H O}$ is the handover probability calculated by real distance from the UE real position to the target microcell;

$P\left(i, j, x_{i}, \hat{x}_{j, p p p}\right)_{H O}, P\left(i, j, x_{i}, \hat{x}_{j, \text { tradition }}\right)_{H O}$ are the handover probability calculated by the predicted distance from the UE predicted position to the target microcell by precise point positioning and standard single point positioning, respectively. 
Table 1. Simulation Parameters

\begin{tabular}{|c|c|c|}
\hline Parameters & Value & Unit \\
\hline Macrocell radius $(\mathrm{r})$ & $20-60$ & $\mathrm{~m}$ \\
\hline Microcell radius $(\mathrm{R})$ & 800 & $\mathrm{~m}$ \\
\hline Macrocell carrier frequency $\left(f_{\text {macro }}\right)$ & 5 & $\mathrm{GHz}$ \\
\hline Microcell carrier frequency $\left(f_{\text {micro }}\right)$ & 900 & $\mathrm{MHz}$ \\
\hline UE velocity $(\mathrm{V})$ & $0-5$ & $\mathrm{~m} / \mathrm{s}$ \\
\hline shadow fading standard deviation $\left(\sigma_{\text {macro }}\right)$ & 4 & $\mathrm{~dB}$ \\
\hline shadow fading standard deviation $\left(\sigma_{\text {micro }}\right)$ & 3 & $\mathrm{~dB}$ \\
\hline Handover threshold $(\Gamma)$ & 3 & $\mathrm{~dB}$ \\
\hline Macrocell transmit power $\left(P t_{\text {macro }}\right)$ & 43 & $\mathrm{dBm}$ \\
\hline Macrocell transmit power $\left(P t_{\text {micro }}\right)$ & 23 & $\mathrm{dBm}$ \\
\hline PPP inherent error $\left(e_{p p p}\right)$ & 0.05 & $\mathrm{~m}$ \\
\hline Single point positioning inherent error $\left(e_{s p}\right)$ & 10 & $\mathrm{~m}$ \\
\hline Measurement interval $(\Delta t)$ & 1 & $\mathrm{~s}$ \\
\hline Simulation time $(\mathrm{t})$ & 10000 & $\mathrm{~s}$ \\
\hline
\end{tabular}

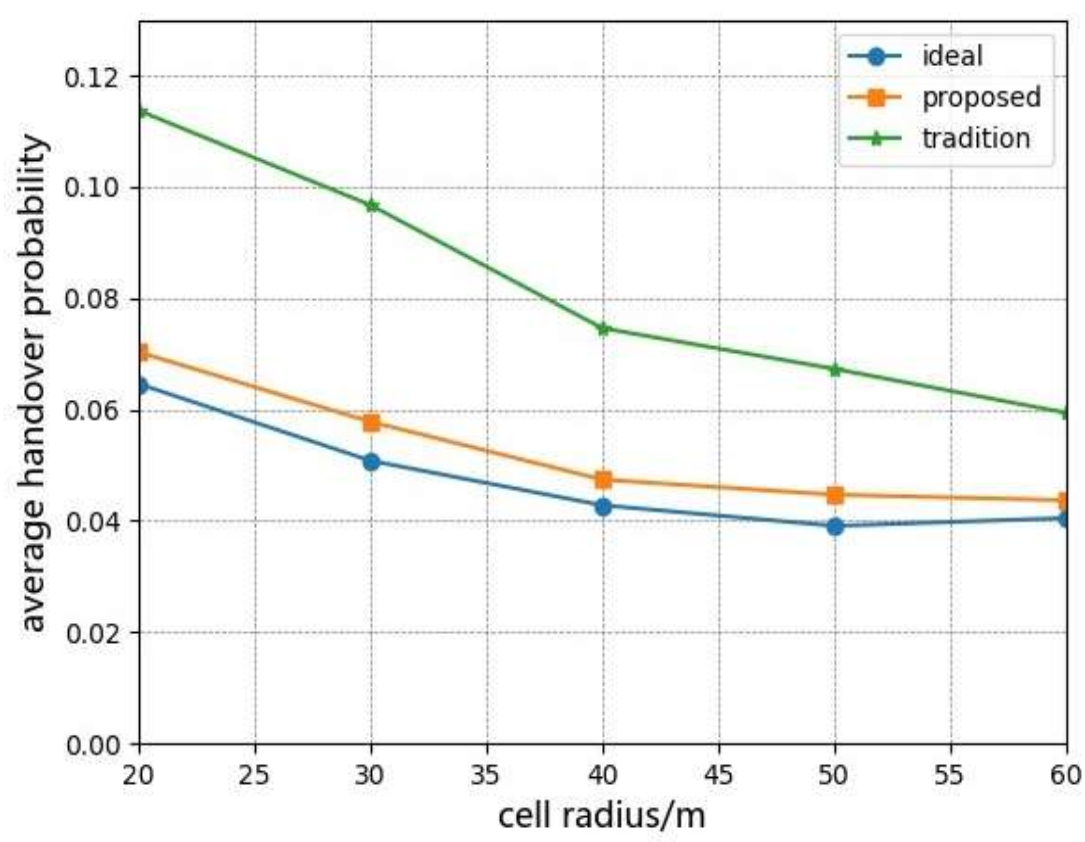

Figure 4. Average Handover Probability

Figure 4 shows the average handover probability when the microcell radius changes from $20 \mathrm{~m}$ to $60 \mathrm{~m}$. It can be seen that as the radius of the microcell increases, the average handover probability decreases. Compared with the traditional way of predicting user position using standard single point positioning, this paper proposes using precise point positioning to predict the user's position, which greatly improves the accuracy of the prediction and reduces the average handover probability. Under the same microcell 
radius, the proposed scheme is superior to the traditional scheme. Under the different microcell radius, handover probability of the proposed scheme changes slower than the traditional scheme, making the system robust.

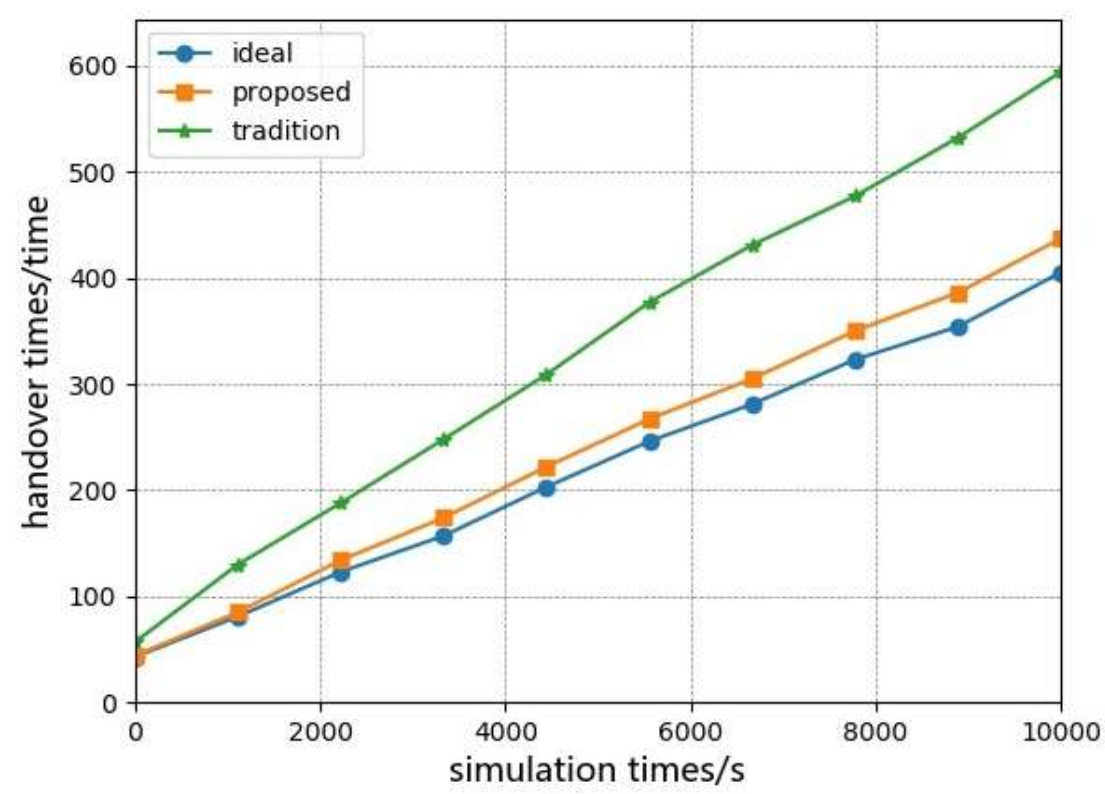

Figure 5. Handover Probability

Figure 5 shows the relationship between simulation time and handover time when the microcell radius is $50 \mathrm{~m}$. It can be seen that as the simulation time increases, the number of handovers continues to increase. Compared with the traditional scheme, the proposed scheme increase rate is obviously lower than the traditional scheme. The non-essential handover is less than the traditional scheme, which shows that the scheme proposed in this paper can reduce the number of unnecessary handovers.

\subsection{System Performance}

In this paper, the Ping-Pong effect and the average delay of the system are used as indicators to evaluate the performance of the system.

3.3.1. Ping-Pong Effect: The Ping-Pong effect is defined as these unnecessary handovers that occur after the handover from the source microcell to the target microcell and handover back to the source microcell in a short time. This kind of unnecessary handover will increase the processing load of the system, and reducing the Ping-Pong effect can greatly reduce the system processing overhead and call drop rate. Ping-Pong handover rate is defined as[16]:

$P_{\text {ping-pongHO }}=N_{\text {ping-pongHO }} / N_{H O}$

Where:

$P_{\text {рing- роng } \mathrm{HO}}$ is the Ping-Pong handover rate of the system;

$N_{\text {ping-pongHO }}$ is the number of Ping-Pong handovers;

$N_{H O}$ is the total handover executions. 
3.3.2. Average Delay: The average delay is defined as the ratio of the total delay to the number of measurements made when switching occurs. As the number of handovers increases, the average delay of the system increases, which leads to a decrease in system throughput and affects the user experience. For the convenience of discussion, the delay of the handover is set to a delay of $50 \mathrm{~ms}$ for a typical LTE user plane. The average delay is defined as:

$$
T_{\text {delay }}=H O_{\text {time }}\left(N_{\text {HO }}-N_{\text {idealHO }}\right) / N_{\text {idealHO }}
$$

where:

$T_{\text {delay }}$ is the average delay of the system;

$H O_{\text {time }}$ is the LTE typical user plane delay;

$N_{\text {idealHo }}$ is the necessary handover times.

Figure 6 shows the impact of the proposed scheme on system performance compared to the traditional scheme. Using the proposed scheme, the Ping-Pong handover rate is $7.32 \%$, and the average delay increase rate is $7.90 \%$. With the traditional scheme, the Ping-Pong handover rate is $31.82 \%$, and the average delay increase rate is $46.67 \%$. It can be seen that, with the proposed scheme, the Ping-Pong handover rate is reduced by $24.50 \%$ and the delay increase rate is decreased by $38.77 \%$ compared with the traditional scheme. Therefore, the proposed scheme in this paper can improve system performance.

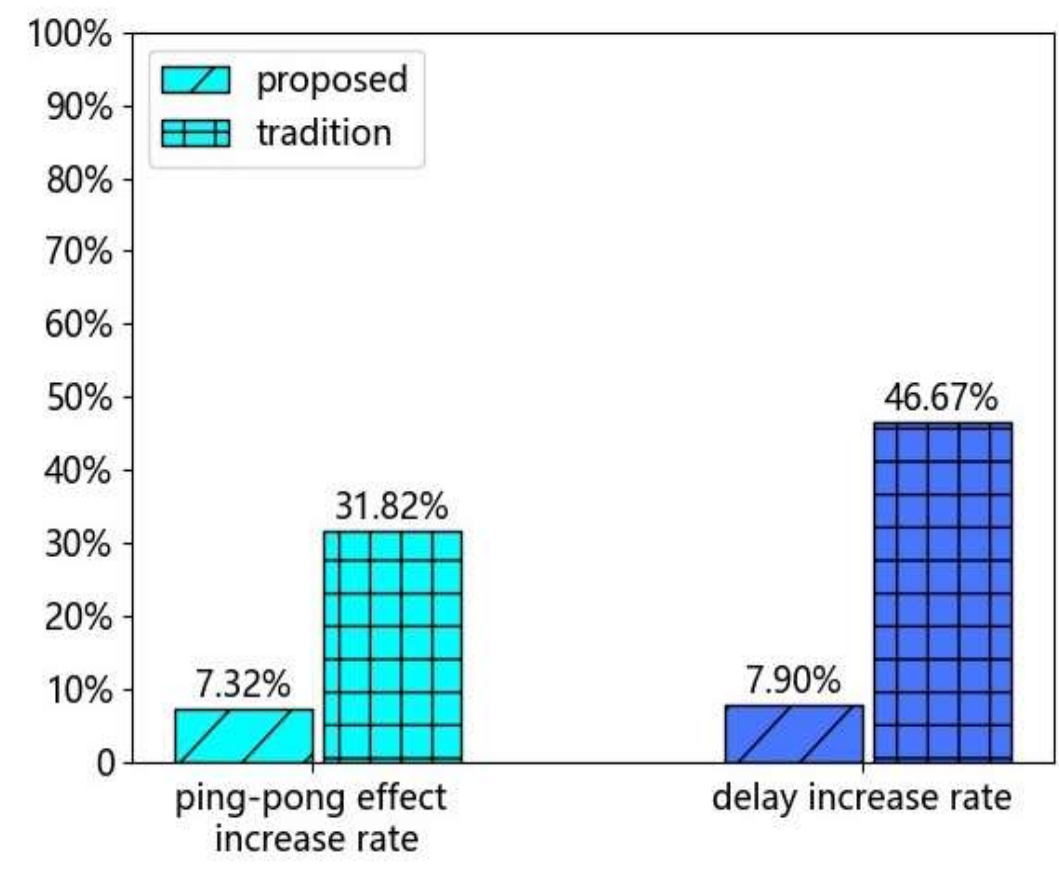

Figure 6. Impact of System Performance

\section{Conclusion}

For 4G, mobility management mainly refers to the management of UE mobility. However, mobility management method in the traditional cellular network is difficult to apply in UDN. In this paper, we proposed a mobility management scheme based on GNSS precise point positioning. The scheme can predict the position of the mobile UE in real time with high accuracy, then calculate the handover trigger indicator for handover decision. We compared through extensive simulations and analysis, with the proposed 
scheme, the handover probability is lower than the traditional approach under different microcell radius. Additionally, our new scheme minimizes the number of unnecessary handovers. Finally, using our proposal can effectively reduce the impact of Ping-Pong effect and the average delay in the system and improve the system performance.

\section{References}

[1] M. Z. Wang Y and Zhang P, "Ultra Dense Networks key technologies and research status", Journal of Beijing University of Posts and Telecommunications, vol. 38, no. 5, (2015), pp. 1-17.

[2] H. Ishii, Y. Kishiyama and H. Takahashi, "A novel architecture for LTE-B :C-plane/U-plane split and Phantom Cell concept", in GLOBECOM Workshops, (2013), pp. 624-630.

[3] K. L. Yeung and S. Nanda, "Channel management in microcell/macrocell cellular radio systems", IEEE Transactions on Vehicular Technology, vol. 45, no. 4, (2002), pp. 601-612.

[4] C. W. Sung and W. S. Wong, "User speed estimation and dynamic channel allocation in hierarchical cellular system", Proc.ieee Veh.tech.conf, vol. 1, (1994), pp. 91-95.

[5] C. D. Wann and Y. M. Chen, "Position tracking and velocity estimation for mobile positioning systems", in The International Symposium on Wireless Personal Multimedia Communications, vol. 1, (2002), pp. 310-314

[6] J. Zhang, J. Feng, C. Liu and X. Hong, "Mobility enhancement and performance evaluation for 5G Ultra dense Networks", Wireless Communications and Networking Conference, (2015), pp. 1793-1798.

[7] K. L. Yap and Y. W. Chong, "Optimized access point selection with mobility prediction using hidden Markov Model for wireless network", Ninth International Conference on Ubiquitous and Future Networks, (2017), pp. 38-42.

[8] A. B. Cheikh, M. Ayari, R. Langar, G. Pujolle and L. A. Saidane, "Optimized Handoff with Mobility Prediction Scheme Using HMM for femtocell networks", IEEE International Conference on Communications, (2015), pp. 3448-3453.

[9] J. Yang, C. Dai and Z. Ding, "A scheme of terminal mobility prediction of Ultra Dense Network based on SVM", 2017 IEEE 2nd International Conference on Big Data Analysis (ICBDA), (2017), pp. 837842.

[10] C. Cai, Y. Gao, L. Pan and J. Zhu, "Precise point positioning with quad-constellations: GPS, BeiDou, GLONASS and Galileo", Advances in Space Research, vol. 56, no. 1, (2015), pp. 133-143.

[11] F. Zhou, D. Dong, W. Li, X. Jiang, J. Wickert and H. Schuh, "GAMP: An open-source software of multi-GNSS precise point positioning using undifferenced and uncombined observations", GPS Solutions, vol. 22, no. 2, (2018).

[12] J. Kouba and P. Héroux, "Precise Point Positioning Using IGS Orbit and Clock Products", Gps Solutions, vol. 5, no. 2, (2001), pp. 12-28.

[13] J. Saastamoinen, "Contributions to the theory of atmospheric refraction", Bulletin Géodésique (19461975), vol. 107, no. 1, (1973), pp. 13-34.

[14] P. Heino "CP5-026 WINNER+ D5.3 v1.0 WINNER+ Final Channel Models", Waseda Commercial Review, vol. 374, (2010).

[15] T. Takasu and A. Yasuda, "Development of the low-cost RTK-GPS receiver with an open source program package RTKLIB", International Symposium on Gps/gnss Jeju South Korea, (2009).

[16] A. Singhrova and N. Prakash, "Adaptive Vertical Handoff Decision Algorithm for Wireless Heterogeneous Networks", IEEE International Conference on High PERFORMANCE Computing and Communications, (2009), pp. 476-481.

\section{Authors}

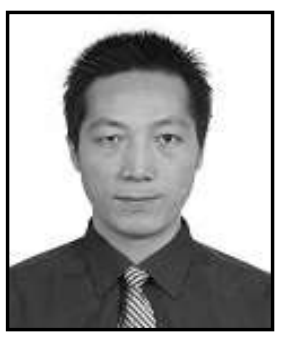

Junxuan Wang, he received the B.E. degree in Northwestern Polytechnical University, China, in 1994, M.E. degree in Xi'an University of Science and Technology, China, in 2002, and Ph.D. degree in Beijing university of Post and Telecommunications, China, in 2005, respectively. He is currently a professor in school of communication engineering, Xi'an University of Post and Telecommunications. His research interests include the areas of $5 \mathrm{G}$ network, wireless communications, etc. 


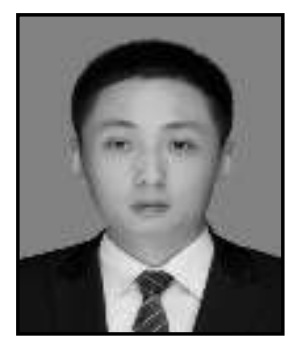

Ruixin Li, he received the B.E. degree in communication engineering from $\mathrm{Xi}^{\prime}$ an University of Post and Telecommunications, Xi'an, China. He is currently working toward the Master degree with the Shaanxi Key Laboratory of Information Communication Network and Security, Xi'an University of post and Telecommunications. His research interests include handover, network architecture, and reliable wireless communication for Ultra Dense Network.

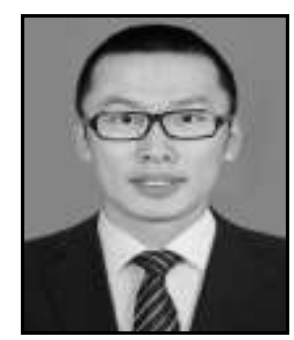

Tianxiang Ji, he received B.S. degree in Electronic Information Science and Technology from Shandong University of Technology in 2016, he is currently studying at the School of Communication and Information Engineering of the University of Posts and Telecommunications. His current main areas of research is the research on inter-cell handover algorithm based on machine learning in $5 \mathrm{G}$ network. 